\title{
Sufficient Conditions of Optimality for Control of Hydro-Electric Power Stations
}

\author{
M.M. A. Ferreira*, A.F. Ribeiro ${ }^{\dagger}$ and G.V. Smirnov** \\ ${ }^{*}$ FEUP, ISR, University of Porto, Portugal \\ ${ }^{\dagger}$ University of Porto, Portugal \\ ** Centre of Physics, Department of Mathematics and Applications, \\ University of Minho, Portugal
}

\begin{abstract}
In this paper we consider a control problem for a cascade of hydro-electric power stations where some of the stations have reversible turbines. The objective of our work is to optimize the profit of power production satisfying restrictions on the water level in the reservoirs. We obtain sufficient conditions of optimality and illustrate them with a simple example.
\end{abstract}

Keywords: optimal control, sufficient conditions of optimality

PACS: $87.55 . \mathrm{de} ; 02.30 . \mathrm{Yy} ; 02.30 . \mathrm{Xx}$

\section{INTRODUCTION}

Water is becoming a scarce resource and this has impact on how the water is used to produce electric energy. The management of multireservoir systems has attracted the attention of many researchers in different contexts [1]. It is especially important if there is also a possibility of reusing the downstream water in a situation of drought. This may be implemented in modern reversible hydro-electric power stations, associated with reservoirs along a river basin with a cascade structure, where it is possible both to turbine water from upstream to produce electric power and to pump from downstream to refill an upstream reservoir. Here we consider a simplified model (based on $[2,3,4,5]$ ) for a cascade of hydro-electric power stations where some of the stations have reversible turbines. The water level in the reservoirs is subject to some constraints. The problem is considered in the framework of optimal control theory. Taking advantage of the particular structure of the problem we prove sufficient conditions of optimality allowing one to show that a given solution is optimal in a local sense. We analytically show an improvement of the profit, due to the use of reversible power stations.

\section{PROBLEM STATEMENT}

For a cascade of $N$ hydro-electric power stations, the dynamics of water volumes, $V_{k}(t)$, in the reservoirs $k=\overline{1, N}$, is described by the following control system

$$
\dot{V}_{k}(t)=A_{k}-u_{k}(t)+\sum_{m \in M(k)} u_{m}(t), \quad t \in[0, T], \quad k=\overline{1, N},
$$

where $M(k)$ is the set of indices for upstream reservoirs immediately before reservoir $k$.

Set $V(\cdot)=\left(V_{1}(\cdot), \ldots, V_{N}(\cdot)\right)$ and $u(\cdot)=\left(u_{1}(\cdot), \ldots, u_{N}(\cdot)\right)$. The controls $u(t)=\left(u_{1}(t), \ldots, u_{N}(t)\right)$ are the turbined/pumped flows of water for reservoirs at time $t$, and $A_{k}$ are the incoming flows, $k=\overline{1, N}$. The equation (1) is called water balance equation and is present in many references (see, e.g. [6]).

The control variables and the water volumes satisfy the following technical constraints :

$$
V_{k}(0)=V_{k}(T), \quad V_{k}(t) \in\left[V_{k}^{m}, V_{k}^{M}\right], \quad u_{k}(t) \in\left[u_{k}^{m}, u_{k}^{M}\right] .
$$

Here $V_{k}^{m}$ and $V_{k}^{M}, k=\overline{1, N}$, stand for the imposed minimum and maximum water volumes, respectively; $u_{k}^{m}$ and $u_{k}^{M}$, $k=\overline{1, N}$, are the imposed minimum and maximum turbined/pumped water flows. The objective is to find optimal controls $\hat{u}_{k}(\cdot)$ and respective volumes $\hat{V}_{k}(\cdot)$, that lead to an optimal profile for the management of water in the system:

$$
\begin{gathered}
\text { maximize } J(u(\cdot), V(\cdot))=\sum_{k=1}^{N} \int_{0}^{T} c(t) u_{k}(t)\left(\frac{V_{k}(t)}{S_{k}}+H_{k}-\frac{V_{j(k)}(t)}{S_{j(k)}}-H_{j(k)}\right) d t . \\
\text { 11th International Conference of Numerical Analysis and Applied Mathematics } 2013 \\
\text { AIP Conf. Proc. 1558, 586-589 (2013); doi: 10.1063/1.4825559 } \\
\text { (c) 2013 AIP Publishing LLC 978-0-7354-1184-5/\$30.00 }
\end{gathered}
$$


Here $c(\cdot)$ is the price of the energy, $H_{k}, k=\overline{1, N}$, are the liquid surface elevations, and $S_{k}, k=\overline{1, N}$, are the areas of the reservoirs. The index $j(k)$ is associated to the (unique) downstream reservoir immediately after reservoir $k$.

Using (1) the cost function can be written as

$$
J(u(\cdot), V(\cdot))=\sum_{k=1}^{N} \int_{0}^{T} c(t)\left(-\dot{V}_{k}(t)+A_{k}+\sum_{m \in M(k)} u_{m}(t)\right)\left(\frac{V_{k}(t)}{S_{k}}+H_{k}-\frac{V_{j(k)}(t)}{S_{j(k)}}-H_{j(k)}\right) d t .
$$

Lemma 1. The following equality holds:

$$
\int_{0}^{T} \sum_{k=1}^{N} c(t)\left(\frac{V_{k}(t)}{S_{k}} \sum_{m \in M(k)} u_{m}(t)-u_{k}(t) \frac{V_{j(k)}(t)}{S_{j(k)}}\right) d t=0
$$

Proof. Since $m \in M(k)$ implies $k \in j(m)$ and $j(k)$ is empty or has only one element, we have

$$
\begin{aligned}
& \int_{0}^{T} \sum_{k=1}^{N} c(t)\left(\frac{V_{k}(t)}{S_{k}} \sum_{m \in M(k)} u_{m}(t)-u_{k}(t) \frac{V_{j(k)}(t)}{S_{j(k)}}\right) d t=\int_{0}^{T} c(t)\left(\sum_{m=1}^{N} \sum_{k \in j(m)} \frac{V_{k}(t)}{S_{k}} u_{m}(t)-\sum_{k=1}^{N} \frac{V_{j(k)}(t)}{S_{j(k)}} u_{k}(t)\right) d t \\
= & \int_{0}^{T} c(t)\left(\sum_{k=1}^{N} \sum_{m \in j(k)} \frac{V_{m}(t)}{S_{m}} u_{k}(t)-\sum_{k=1}^{N} \frac{V_{j(k)}(t)}{S_{j(k)}} u_{k}(t)\right) d t=\int_{0}^{T} c(t)\left(\sum_{k=1}^{N} \frac{V_{j(k)}(t)}{S_{j(k)}} u_{k}(t)-\sum_{k=1}^{N} \frac{V_{j(k)}(t)}{S_{j(k)}} u_{k}(t)\right) d t=0 .
\end{aligned}
$$

\section{SUFFICIENT CONDITIONS OF OPTIMALITY}

We shall use the notation $B V([0, T], R)$ for the space of bounded variation function $f:[0, T] \rightarrow R$. We assume that $c(\cdot) \in B V([0, T], R), c(\cdot)$ is right continuous and $c(0)=c(T)$.

Without changing the notation for the cost function $J$, we convert the maximization problem into a minimization one. Integrating (2) by parts and using Lemma 1 we obtain the following problem:

$$
\begin{gathered}
\text { minimize } J(u(\cdot), V(\cdot))= \\
=-\sum_{k=1}^{N}\left[\frac{A_{k}}{S_{k}} \int_{0}^{T} c(t) V_{k}(t) d t+\left(H_{k}-H_{j(k)}\right) \int_{0}^{T} \frac{d c(t)}{d t}\left(V_{k}(t)+\sum_{m \in \mathscr{M}(k)} V_{m}(t)\right) d t+\frac{1}{2 S_{k}} \int_{0}^{T} \frac{d c(t)}{d t} V_{k}^{2}(t) d t\right], \\
\dot{V}_{k}(t)=A_{k}-u_{k}(t)+\sum_{m \in M(k)} u_{m}(t), \\
V_{k}(0)=V_{k}(T), \quad V_{k}(t) \in\left[V_{k}^{m}, V_{k}^{M}\right], \quad u_{k}(t) \in\left[u_{k}^{m}, u_{k}^{M}\right] .
\end{gathered}
$$

The set $\mathscr{M}(k)$ contains the indices corresponding to all upstream reservoirs appearing in cascade before reservoir $k$.

Theorem 1. Let $\left(\hat{u}_{k}(\cdot), \hat{V}_{k}(\cdot)\right), k=\overline{1, N}$, be a control process. Assume that the following conditions are satisfied:

1. there exist functions $p_{k}(\cdot) \in B V([0, T], R)$ and $\mu_{k}(\cdot) \in B V([0, T], R), k=\overline{1, N}$, satisfying

$$
\begin{gathered}
\frac{d p_{k}(t)}{d t}=-\frac{A_{k}}{S_{k}} c(t)-\left(H_{k}-H_{j(k)}\right) \frac{d c(t)}{d t}-\sum_{l \in \mathscr{M}^{-1}(k)}\left(H_{l}-H_{j(l)}\right) \frac{d c(t)}{d t}-\frac{\hat{V}_{k}(t)}{S_{k}} \frac{d c(t)}{d t}+\frac{d \mu_{k}}{d t}, \\
p_{k}(0)=p_{k}(T) ;
\end{gathered}
$$

2. the equality $\max _{u_{k} \in\left[u_{k}^{m}, u_{k}^{M}\right], k=\overline{1, N}} \sum_{k=1}^{N} p_{k}(t)\left(-u_{k}+\sum_{m \in \mathscr{M}(k)} u_{m}\right)=\sum_{k=1}^{N} p_{k}(t)\left(-\hat{u}_{k}(t)+\sum_{m \in \mathscr{M}(k)} \hat{u}_{m}(t)\right)$, holds;

3. the functions $\mu_{k}(\cdot), k=\overline{1, N}$, satisfy the inequalities

$$
\left.\frac{d \mu_{k}(t)}{d t} \leq 0, \text { if } \quad \hat{V}_{k}(t)=V_{k}^{m} ; \quad \frac{d \mu_{k}(t)}{d t} \geq 0, \text { if } \quad \hat{V}_{k}(t)=V_{k}^{M} ; \quad \frac{d \mu_{k}(t)}{d t}=0, \text { if } \quad \hat{V}_{k}(t) \in\right] V_{k}^{m}, V_{k}^{M}[;
$$


4. if $d c(t) / d t>0$, then the functions $\mu_{k}(\cdot), k=\overline{1, N}$, satisfy the inequalities

$$
\frac{d \mu_{k}(t)}{d t}<0, \text { if } \quad \hat{V}_{k}(t)=V_{k}^{m} ; \quad \frac{d \mu_{k}(t)}{d t}>0 \text {, if } \quad \hat{V}_{k}(t)=V_{k}^{M} ;
$$

5. if $\hat{V}(t) \in] V^{m}, V^{M}[$, then $d c(t) / d t \leq 0$.

Then $J(\hat{u}(\cdot)+h \bar{u}(\cdot), \hat{V}(\cdot)+h \bar{V}(\cdot)) \geq J(\hat{u}(\cdot), \hat{V}(\cdot))$ wherever $\left(\hat{u}_{k}(\cdot)+\bar{u}_{k}(\cdot), \hat{V}_{k}(\cdot)+\bar{V}_{k}(\cdot)\right), k=\overline{1, N}$, is an admissible process and $h>0$ is sufficiently small.

Proof. Let $\left(\hat{u}_{k}(\cdot)+\bar{u}_{k}(\cdot), \hat{V}_{k}(\cdot)+\bar{V}_{k}(\cdot)\right), k=\overline{1, N}$, be an admissible process, and let $h>0$ be sufficiently small. Then we have

$$
\begin{aligned}
\Delta J=J(\hat{u}(\cdot)+h \bar{u}(\cdot), \hat{V}(\cdot)+h \bar{V}(\cdot))-J(\hat{u}(\cdot), \hat{V}(\cdot))= & -\sum_{k=1}^{N}\left[h \int_{0}^{T}\left(\frac{A_{k}}{S_{k}} c(t)+\left(\left(H_{k}-H_{j(k)}\right)+\frac{\hat{V}_{k}(t)}{S_{k}}\right) \frac{d c(t)}{d t}\right) \bar{V}_{k}(t) d t\right. \\
& \left.+h \sum_{m \in \mathscr{M}(k)}\left(H_{k}-H_{j(k)}\right) \int_{0}^{T} \frac{d c(t)}{d t} \bar{V}_{m}(t) d t+\frac{h^{2}}{2 S_{k}} \int_{0}^{T} \frac{d c(t)}{d t} \bar{V}_{k}^{2}(t) d t\right] .
\end{aligned}
$$

Using (3) we get

$$
\begin{aligned}
\Delta J & =\sum_{k=1}^{N}\left[h \int_{0}^{T}\left(\frac{d p_{k}(t)}{d t}+\sum_{l \in \mathscr{M}^{-1}(k)}\left(H_{l}-H_{j(l)}\right) \frac{d c(t)}{d t}-\frac{d \mu_{k}(t)}{d t}\right) \bar{V}_{k}(t) d t\right. \\
& \left.-h \sum_{m \in \mathscr{M}(k)}\left(H_{k}-H_{j(k)}\right) \int_{0}^{T} \frac{d c(t)}{d t} \bar{V}_{m}(t) d t-\frac{h^{2}}{2 S_{k}} \int_{0}^{T} \frac{d c(t)}{d t} \bar{V}_{k}^{2}(t) d t\right] .
\end{aligned}
$$

Observe that

$$
\sum_{k=1}^{N} \sum_{l \in \mathscr{M}^{-1}(k)}=\sum_{(l, k) \in \operatorname{grph} \mathscr{M}}=\sum_{l=1}^{N} \sum_{k \in \mathscr{M}(l)}
$$

From this we obtain

$$
\Delta J=\sum_{k=1}^{N}\left[h \int_{0}^{T}\left(\frac{d p_{k}(t)}{d t}-\frac{d \mu_{k}(t)}{d t}\right) \bar{V}_{k}(t) d t-\frac{h^{2}}{2 S_{k}} \int_{0}^{T} \frac{d c(t)}{d t} \bar{V}_{k}^{2}(t) d t\right] .
$$

Integrating by parts and using periodicity conditions we get

$$
\Delta J=\sum_{k=1}^{N}\left[h \int_{0}^{T} p_{k}(t)\left(\bar{u}_{k}(t)-\sum_{m \in \mathscr{M}(k)} \bar{u}_{m}(t)\right) d t-h \int_{0}^{T} \bar{V}_{k}(t) \frac{d \mu_{k}(t)}{d t} d t-\frac{h^{2}}{2 S_{k}} \int_{0}^{T} \frac{d c(t)}{d t} \bar{V}_{k}^{2}(t) d t\right] \geq 0 .
$$

\section{SPECIAL CASE}

For illustration, consider the case of one power station with single incoming flow $A$. Let $\tau, c_{1}, c_{2}$ be constants such that $\tau \in] 0, T\left[\right.$ and $c_{1}<c_{2}$. Take $c(t)= \begin{cases}c_{1}, & t \in[0, \tau[, \\ c_{2}, & t \in[\tau, T[, \\ c_{1}, & t=T .\end{cases}$

$\Delta J=J(\hat{u}(\cdot)+h \bar{u}(\cdot), \hat{V}(\cdot)+h \bar{V}(\cdot))-J(\hat{u}(\cdot), \hat{V}(\cdot))=h \int_{0}^{T} p(t) \bar{u}(t) d t-h \int_{0}^{T} \bar{V}(t) \frac{d \mu(t)}{d t} d t+h^{2} \frac{c_{2}-c_{1}}{2 S}\left(\bar{V}^{2}(0)-\bar{V}^{2}(\tau)\right)$.

Let $u_{\min }<0<A<u_{\max }$ and $V_{\min }<V_{\max }-\theta\left(A-u_{\min }\right)$, where $\theta=\frac{u_{\max }-A}{A-u_{\min }}(T-\tau)$. Assume that $\theta<\tau$ and consider the process $(\hat{u}(\cdot), \hat{V}(\cdot))$, where

$$
\hat{u}(t)=\left\{\begin{array}{ll}
u_{\min }, & t \in[0, \theta], \\
A, & t \in] \theta, \tau], \\
u_{\max }, & t \in] \tau, T],
\end{array} \quad \text { and } \quad \hat{V}(t)= \begin{cases}V_{\max }+(t-\theta)\left(A-u_{\min }\right), & t \in[0, \theta], \\
V_{\max }, & t \in] \theta, \tau], \\
V_{\max }+(t-\tau)\left(A-u_{\max }\right), & t \in] \tau, T]\end{cases}\right.
$$


Consider also the functions

$$
\mu(t)=\left\{\begin{array}{ll}
0, & t \in[0, \theta[, \\
c_{1}(t-\theta) A / S, & t \in[\theta, \tau[, \\
c_{1}(\tau-\theta) A / S+\Delta \mu, & t \in[\tau, T],
\end{array} \quad \text { and } \quad p(t)= \begin{cases}(\theta-t) c_{1} A / S, & t \in[0, \theta], \\
0, & t \in \theta, \tau[, \\
p_{\tau}-(t-\tau) c_{2} A / S, & t \in[\tau, T[, \\
p(T)=\theta c_{1} A / S, & t=T,\end{cases}\right.
$$

where $\Delta \mu=\theta \frac{c_{1} A}{S}+(T-\tau) \frac{c_{2} A}{S}+\frac{c_{2}-c_{1}}{S}(\hat{V}(\tau)-\hat{V}(0))$ and $p_{\tau}=-\left(c_{2}-c_{1}\right) H-\frac{c_{2}-c_{1}}{S} \hat{V}(\tau)+\Delta \mu$. If $p_{\tau} \leq 0$, then, since $\bar{V}(\tau) \leq 0$ and $\Delta \mu>0$, we have

$$
\Delta J \geq-h \Delta \mu \bar{V}(\tau)-h^{2} \frac{c_{2}-c_{1}}{2 S} \bar{V}^{2}(\tau) \geq 0
$$

whenever $\hat{V}(\cdot)+\bar{V}(\cdot)$ is admissible and $h>0$ is sufficiently small.

Example 1. Let $A=1, T=12, \tau=6, c_{1}=2, c_{2}=5, u_{\min }=-1, u_{\max }=2, V_{\min }=3, V_{\max }=10, S=100$. Then $\hat{V}(0)=4$ and $\theta=3$. The condition $p_{\tau}<0$ is satisfied. The optimal process is shown in Fig. 1.

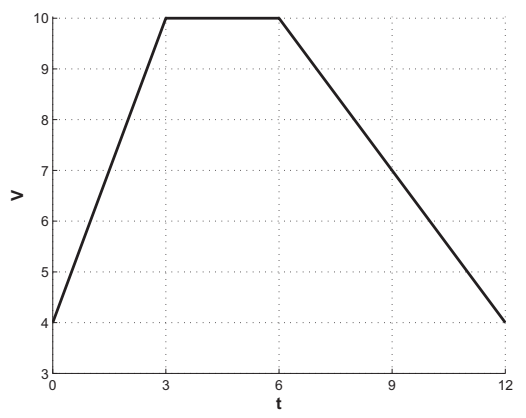

FIGURE 1. Optimal process

Note that $\hat{u}(t)=-1, t \in[0,3]$, i.e. the water is pumped. In this way the station accumulates water when the price is low to be used when the price is higher. Therefore the use of reversible turbines improves the profit.

\section{ACKNOWLEDGMENTS}

This work has been partially supported by the European Union Seventh Framework Programme [FP7-PEOPLE-2010ITN] under grant agreement n. 64735-SADCO and FCT project PTDC/EEA-CRO/116014/2009.

\section{REFERENCES}

1. J. W. Labadie, Optimal Operation of multireservoir systems: state-of-the-art review, J. Water Resour. Plng. and Mgmt., 130(2), pp.93-111 (2004)

2. Andrei Korobeinikov, Alexander Kovacec, Mark McGuinness, Marta Pascoal, Ana Pereira and Sónia Vilela, Optimizing the profit from a complex cascade of hydroelectric stations with recirculating water, Mathematics-in-industry case studies journal, 2, pp.111-133 (2010)

3. International Atomic Energy Agency, Valoragua - A model for the optimal operating strategy of mixed hydrothermal generating systems, Users' manual for the mainframe computer version, IAEA computer manual series no. 4, Vienna (1992)

4. R. Liang and Y. Hsu, Hydroelectric generation scheduling using self-organizing feature maps, Electric Power Systems Research, 30 (1), pp.1-8 (1994)

5. A.F. Ribeiro, M.C.M. Guedes, G.V. Smirnov and S. Vilela, On the optimal control of a cascade of hydro-electric power stations, Electric Power Systems Research., 88, pp.121-129 (2012)

6. S.J.P. Mariano, J. P. S. Catalao, V. M. F. Mendes, and L.A.F.M. Ferreira, Profit-Based Short-Term Hydro Scheduling considering Head-Dependent Power Generation, Power Tech, 2007 IEEE Lausanne, pp.1362-1367 (July 2007) 